\title{
Exploring CTCF and cohesin related chromatin architecture at HOXA gene cluster in primary human fibroblasts
}

\author{
WANG Xing, XU Miao, ZHAO GuangNian, LIU GuoYou, HAO DeLong, LV Xiang ${ }^{*}$ \\ \& LIU DePei* \\ State Key Laboratory of Medical Molecular Biology, Department of Biochemistry and Molecular Biology, Institute of Basic Medical Sciences, \\ Chinese Academy of Medical Sciences \& Peking Union Medical College, Beijing 100005, China
}

\begin{abstract}
Spatial expression patterns of homeobox $(H O X)$ genes delineate positional identity of primary fibroblasts from different topographic sites. The molecular mechanism underlying the establishing or maintaining of HOX gene expression pattern remains an attractive developmental issue to be addressed. Our previous work suggested a critical role of CTCF/cohesin-mediated higher-order chromatin structure in RA-induced HOXA activation in human teratocarcinoma NT2/D1 cells. This study investigated the recruitment of CTCF and cohesin, and the higher-order chromatin structure of the HOXA locus in fetal lung and adult foreskin fibroblasts, which display complementary HOXA gene expression patterns. Chromatin contacts between the CTCF-binding sites were observed with lower frequency in human foreskin fibroblasts. This observation is consistent with the lower level of cohesin recruitment and 5' HOXA gene expression in the same cells. We also showed that CTCF-binding site A56 (CBSA56) related chromatin structures exhibit the most notable changes in between the two types of cell, and hence may stand for one of the key CTCF-binding sites for cell-type specific chromatin structure organization. Together, these results imply that $\mathrm{CTCF} /$ cohesin coordinates $\mathrm{HOXA}$ cluster higher-order chromatin structure and expression during development, and provide insight into the relationship between cell-type specific chromatin organization and the spatial collinearity.
\end{abstract}

human fibroblasts, $\mathrm{HOXA}$ cluster, higher-order chromatin structure, CTCF, cohesion

Citation: Wang X, Xu M, Zhao GN, Liu GY, Hao DL, Lv X, Liu DP. Exploring CTCF and cohesin related chromatin architecture at HOXA gene cluster in primary human fibroblasts. Sci China Life Sci, 2015, 58: 860-866, doi: 10.1007/s11427-015-4913-5

Cell-specific gene expression profiles are related to their relative anatomic positions (anterior-posterior, proximal-distal, and ventral-dorsal) [1,2]. Fidelity of $H O X$ expression pattern specifies the proper positional identity in cells and the normal homeostasis of tissues and organs [3]. In each topographic site, the unique "HOX code" leads to site-specific cellular differentiation and tissue morphogenesis [2]. The $H O X$ genes are usually organized into clusters on vertebrate chromosome [4]. Clustering is thought to bring about long-range or local regulatory elements to allow $H O X$ gene expression in a more coordinated way $[5,6]$.

*Corresponding author (email: lvxiang@pumc.edu.cn; liudp@pumc.edu.cn)
Specifically, HOX clusters show a particular spatially and temporally collinear expression pattern during development, wherein the domains and sequence of $H O X$ gene activation follow their gene order along the chromosome [7]. Activation of the cluster during development has been interpreted as a multistep process, involving the progressive release of the coding units from heterochromatic silencing [8,9]. HOX loci also undergo complex changes in chromatin structures and long-range chromatin interactions during cellular differentiation [10-12] and embryonic development [13,14]. In the process of colinear activation, $H O X$ clusters switch from a single repressive domain to a bimodal 3D structure, in which transcriptionally active compartment formed by 
clustering of newly activated genes [13].

CCCTC-binding factor (CTCF) is a highly conserved zinc finger protein implicated in diverse regulatory events. Previous studies suggest that CTCF predominantly functions in mediating inter- and intra-chromosomal loops for chromatin domain segregation or long-range gene regulation [15]. CTCF usually works together with cohesin to facilitate long range interactions in the genome, and CTCF is necessary for recruiting of cohesin to their shared binding sites [16,17]. Multiple CTCF binding sites (CBSs) were identified in human $H O X$ clusters, most of which belong to the highly conserved noncoding elements. Our previous work revealed that CTCF functions as a controller of HOXA cluster silencing and mediates polycomb repressive complex 2 (PRC2)-repressive higher-order chromatin structure in NTERA-2 cl.D1 human teratocarcinoma (NT2/D1) cells [18]. Fibroblasts are principal component of human body and have been considered to be very important in wound healing [19] and reprogramming [20]. Differential expression of $H O X$ genes in adult fibroblasts faithfully reflects their position along the anatomic axes of the developing body, and therefore provides a native and convenient model for examining $H O X$ gene regulation. To elucidate whether the $\mathrm{CTCF} /$ cohesin-mediated chromatin architecture represents a general role in the regulation of HOXA genes, we investigated CTCF and cohesin recruitment, and higher order structure of this locus in the primary fetal lung and foreskin fibroblasts that exhibiting complementary patterns of HOXA gene expression [21].

We showed in this study that CTCF/cohesin bound to the CBSs in HOXA locus in both human primary lung and foreskin fibroblasts, and that the CBSs are spatially related as have been indicated in the NT2/D1 cells. The cell-type-specific enrichment of cohesin coincides with gene expression pattern and 3D organization of HOXA cluster in these cells, implying that $\mathrm{CTCF} /$ cohesin also participates in regulating HOXA locus expression and spatial structure in primary human cells. Moreover, we found that CTCF-binding site A56 (CBSA56) likely plays a central role in the spatial organization of HOXA locus, as it gives out the highest and most frequent interactions with other elements in the locus in the $3 \mathrm{C}$ assay.

\section{Materials and methods}

\subsection{Cell culture}

Primary human foreskin fibroblast (FsF) was derived from adult human foreskins (kindly provided by Skin Laboratory of Peking Union Medical College Hospital). FsF and 293T cells were cultured in dulbecco's modified eagle medium (DMEM; Cat. No. 11960, Gibco, USA) supplemented with $3.75 \mathrm{~g} \mathrm{~L}^{-1}$ sodium pyruvate, $10 \%$ fetal bovine serum, $100 \mathrm{U}$ $\mathrm{mL}^{-1}$ penicillin, $100 \mu \mathrm{g} \mathrm{mL}^{-1}$ streptomycin, and $2 \mathrm{mmol} \mathrm{L}^{-1}$
L-glutamine. Primary human fetal lung fibroblasts (HLF) were obtained from Cell Applications (Cat. No. 506-05f, USA), and maintained with HLF growth medium and HLF growth supplement (Cell Applications) according to manufacturer's instructions.

\subsection{Quantitative RT-PCR and real-time PCR}

Quantitative RT-PCR assays were performed in three independent cell cultures. Trizol reagent (Invitrogen, USA) was used to extract total RNA from the cells as described by the manufacturer. Two micrograms of total RNA was treated with DNase I and then was used in each reverse transcription reaction with M-MuLV Reverse Transcriptase (NEB, USA) according to the manufacturer's instructions. After reverse transcription, the $20 \mu \mathrm{L}$ sample volumes were diluted 10 times with TE buffer, and $3 \mu \mathrm{L}$ of diluted cDNA was used for each quantitative PCR reaction. Real-time PCR was performed with the Perfect Real Time Kit (TaKaRa, Dalian) using an thermal cycler (iCycler, Bio-Rad, USA) and iQ5 real-time PCR detection system. Two-step PCR was conducted for 40 cycles, with denaturation at $95^{\circ} \mathrm{C}$ for $5 \mathrm{~s}$ and annealing/extension at $60^{\circ} \mathrm{C}$ for $30 \mathrm{~s}$. The HOXA gene expression was analyzed with the iQ5 software, and normalized to the expression of house-keeping gene glyceraldehyde-3-phosphate dehydrogenase $(G A P D H)$. Primer sets used in RT-PCR, chromosome conformation capture (3C) and chromatin immunoprecipitation (ChIP) assays were described previously [18].

\subsection{Chromosome conformation capture (3C)}

The 3C assay was performed as previously described [18]. Briefly, restricted enzyme $B g l \mathrm{II}$ (NEB, R0144M) was used to digest the $1.7 \%$ formaldehyde-cross-linked nuclei from FsF or HLF cells. The efficiency of BglII digestion was assessed by real-time PCR amplification of digested and undigested DNA fragments that spanned the BglII site, to ensure that more than $80 \%$ of each individual restriction site was digested. The subsequent ligation was performed at DNA concentrations of less than $2.5 \mathrm{ng} \mu \mathrm{L}^{-1}$ with 2 cohesive end units $\mu \mathrm{L}^{-1}$ of T4 DNA ligase (NEB, M0202M) at $16^{\circ} \mathrm{C}$ for $4 \mathrm{~h}$. After reversing the cross-links, the genomic DNA was purified by phenol extraction and ethanol precipitation, and 50-200 ng of the precipitated DNA was analysed by real-time PCR. The DNA of human HOXA bacterial artificial chromosomes (BAC) clone (CH17-233M10) was digested and ligated, to construct a control library. The cross-linking frequencies between the leader fragments and the rest of the HOXA locus were normalized to the frequencies between the same fragments in control library and those between two BglII fragments in the human ERCC3 locus. Owing to the relatively frequent but nonfunctional interactions between neighboring restriction fragments, the signals between several leader fragments and their neigh- 
boring fragments were not detected. All PCR was repeated three times, and all assays were performed in at least three independent cultures.

\subsection{Chromatin immunoprecipitation (ChIP)}

ChIP analysis was carried out in FsF and HLF cells. In brief, isolated, $1 \%$ formaldehyde-cross-linked cells were lysed in lysis buffer (1\% sodium dodecyl sulfate (SDS), 10 mmol L $\mathrm{L}^{-1}$ ethylene diamine tetraacetic acid (EDTA), 50 mmol L ${ }^{-1}$ Tris, $\mathrm{pH}$ 8.1) containing protease inhibitors and sonicated on ice until cross-linked chromatin DNA was sheared to a range of 200-1,000 bp. The sonicated cell supernatant was diluted 10-fold in ChIP dilution buffer $(1.2$ mmol L ${ }^{-1}$ EDTA, $16.7 \mathrm{mmol} \mathrm{L}^{-1}$ Tris- $\mathrm{HCl}, \mathrm{pH} 8.1,0.01 \%$ sodium dodecyl sulfate (SDS), 1.1\% Triton X-100, 167 mmol $\mathrm{L}^{-1} \mathrm{NaCl}$ ). The precleared chromatin using protein G-agarose (Upstate) was incubated with anti-CTCF antibody (Millipore, 07-729, Germany) or anti-RAD21 antibody (Abcam, ab992, UK) at $4{ }^{\circ} \mathrm{C}$ overnight, and a mock immunoprecipitation with rabbit IgG was carried out in parallel. Immunoprecipitates were recovered by incubation with protein $\mathrm{G}$-agarose (Upstate) at $4^{\circ} \mathrm{C}$ for $2 \mathrm{~h}$, followed by low-speed centrifugation. The washed pellets were reverse cross-linked. The DNA was extracted with phenolchloroform-isoamyl alcohol (25:24:1 [vol/vol/vol]), precipitated with ethanol, and used for real-time PCR analysis. Quantitation of PCR products was normalized by comparison to a 1:25 diluted input DNA. All ChIP experiments were performed at least three times with independent cell cultures.

\section{Results}

\subsection{Fetal lung and adult foreskin fibroblasts display complementary $\mathrm{HOXA}$ gene expression patterns}

To highlight different $H O X$ expression patterns that distinguish fibroblast types, we used fetal lung fibroblasts and adult foreskin fibroblasts to represent two distinct positional identities: anterior and visceral vs. posterior and dermal sites. We examined the HOXA cluster gene expression therein by quantitative RT-PCR, and found that human fetal lung and foreskin fibroblasts displayed complementary expression patterns, 3' HOXA genes (HOXA1-HOXA7) were mainly expressed in the fetal lung fibroblasts, while $5^{\prime}$ HOXA genes (HOXA9-HOXA13) were mainly expressed in foreskin fibroblasts (Figure 1). These results consistent with previous observations [21], and suggest that spatial-specific expression pattern of HOXA cluster genes may underlie the detailed positional memory in fibroblast. Besides their known differences in chromatin modifications at the cluster [21], the higher-order chromatin structure of HOXA cluster in fetal lung fibroblasts and adult foreskin fibroblasts, particularly, the CTCF-related higher-order chromatin structure, may also contribute to the differential transcriptional control of HOXA genes in the two cell types.

\subsection{CTCF binding site CBSA56 contributes to cell-type specific chromatin organization of the $H O X A$ cluster in primary human fibroblasts}

We then explored the spatial organization of $H O X A$ cluster in the two primary fibroblasts by $3 \mathrm{C}$ assay. The ligation

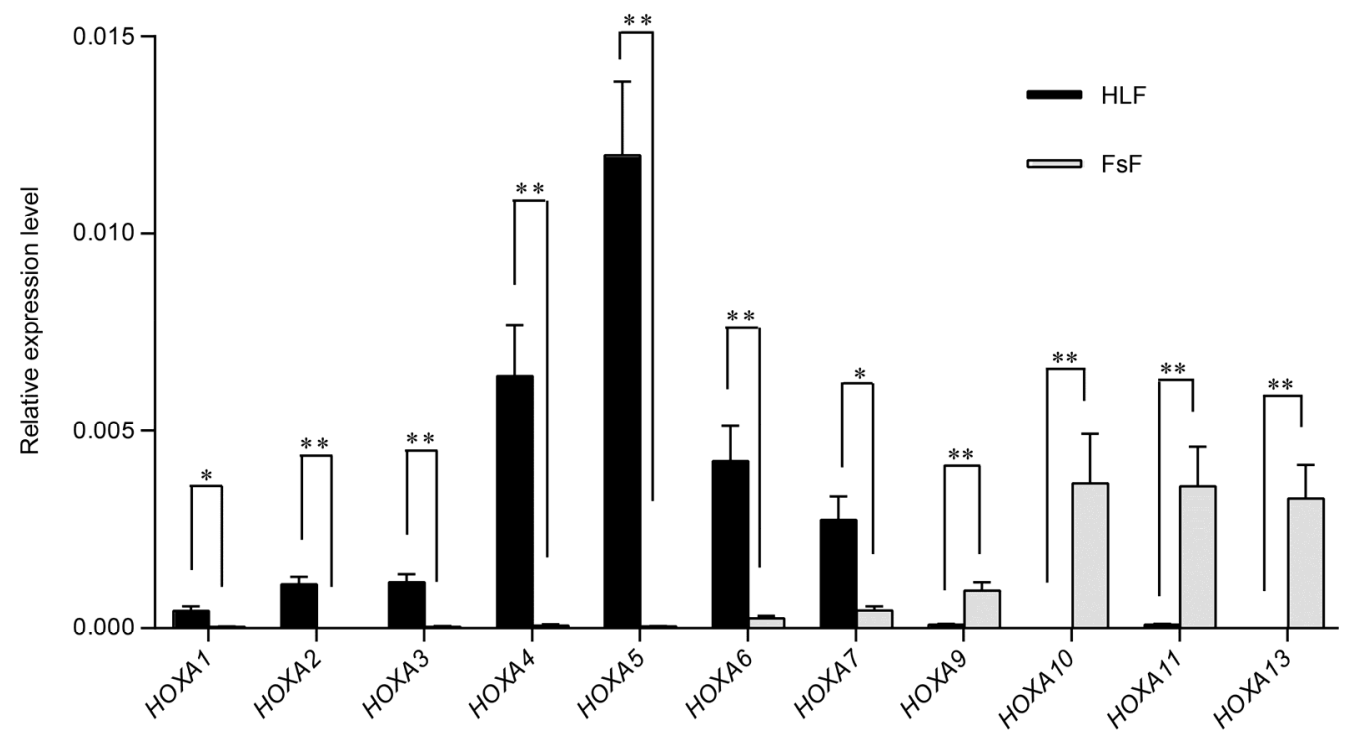

Figure 1 Complementary expression patterns of HOXA genes in human fetal lung fibroblasts (HLF) and foreskin fibroblasts (FsF). HOXA1-HOXA7 were mainly expressed in the fetal lung fibroblasts, while HOXA9-HOXA13 were mainly expressed in foreskin fibroblasts. The HOXA gene expression was determined by quantitative RT-PCR and normalized to GAPDH. Error bars represent standard errors of the means (SEM) of three independent cell cultures. **, $P<0.01 ; *, P<0.05$ (student's $t$-test). 
frequency of a total of $18 \mathrm{Bg} l \mathrm{II}$ fragments containing the nine predicted CTCF-binding sites (CBSs), HOXA gene promoters or retinoic acid response elements (RAREs) were measured. Five leader fragments were chosen to explore the chromatin interactions between the leader fragments and others, these include three CBSs-containing fragments (CBSA56/A5P F8; CBSA79/A7P F11; CBSA10b/A10bP F14) which exhibited strong interactions with other fragments in our previous work [18], and the two border fragments (RARE1 F1 and CBSA13E2/E3 F18). For the first time, we observed strong interactions between fragments containing CBSs in primary human fibroblasts (Figure 2), suggesting that CTCF and its binding sites may also play a critical structural role in the HOXA cluster organization in primary human fibroblasts. Moreover, significant decreases in the relative cross-linking frequencies of three leader fragment $(\mathrm{F} 8, \mathrm{~F} 11$ or F14) to the rest of the cluster were detected in foreskin fibroblasts, compared with the corre- sponding signals in the fetal lung fibroblasts. The CBScentered higher-order chromatin conformation of the HOXA cluster display a more "closed" chromatin conformation in fetal lung fibroblasts than that in foreskin fibroblasts, consistent to the repression of HOXA9-HOXA13 genes in fetal lung fibroblasts. Specifically, in both types of cells, the leader fragment CBSA56/A5P (F8) interacts extensively to the examined DNA fragments throughout the HOXA cluster, including the most CBSs in the cluster and the HOXA1HOXA4 gene promoter elements, suggesting that this region might be located at the center of higher-order chromatin structure of the HOXA cluster in primary cells. This observation is consistent with our previous results in NT2/D1 cells [18], and suggesting that CBSA56 may be one of the key CBSs for chromatin structure organization of the HOXA cluster. The interactions between CBSA56/A5 promoter and other fragments were also significantly decreased in foreskin than that in fetal lung fibroblasts.

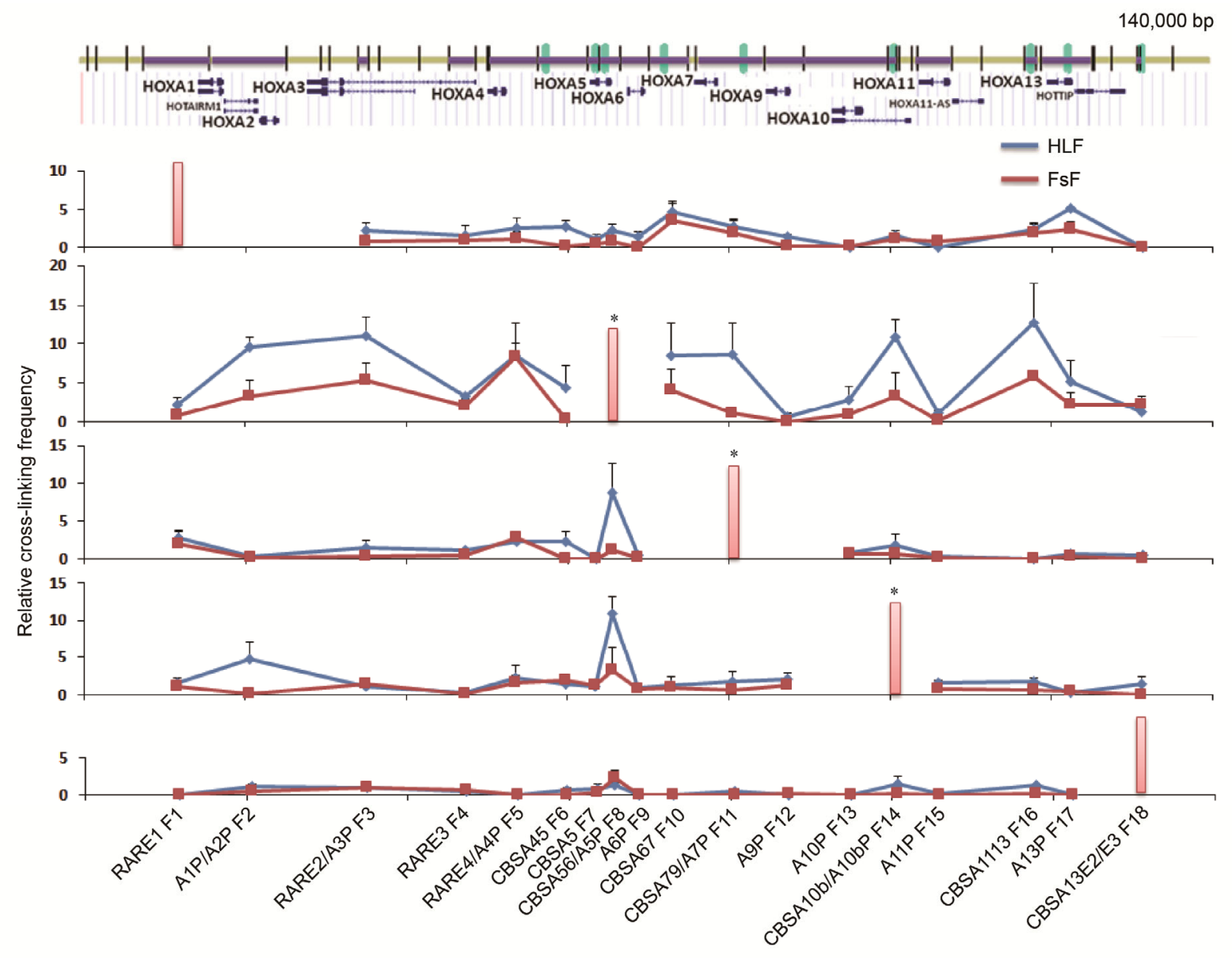

Figure 2 CTCF contributes to higher-order chromatin structure of HOXA locus in human fetal lung fibroblasts (HLF) and foreskin fibroblasts (FsF). Black vertical lines indicate $B g l I I$ sites, and cyan vertical bars indicate predicted CTCF-binding sites (CBSs). The CBSs were named after the nearby $H O X A$ genes, e.g., CBSA45 represents the CBS site between HOXA4 and HOXA5. A total of eighteen BglII fragments (purple horizontal bars) containing the CBSs, HOXA gene promoters or retinoic acid response elements (RAREs) were analyzed by 3C assay. Three CBSs-containing fragments (CBSA56/A5P F8; CBSA79/A7P F11; CBSA10b/A10bP F14) which exhibited strong interactions with other fragments in our previous work [18], and the two border fragments (RARE1 F1 and CBSA13E2/E3 F18) were chosen as leader fragments (pink vertical bars). CBSA56-related chromatin structures exhibited the most notable changes in between the two types of cell. The SEM of the triplicate experiments is indicated. *, $P<0.05$ (student's $t$-test for the population mean of the $3 \mathrm{C}$ signals of each leader fragment in the two cell types). 
To determine whether the CBS-relevant higher-order chromatin conformation is generally applied to the HOXA locus, we explored the spatial organization of the locus in
293 T cells (Figure 3A), and observed a chromatin conformation similar to that in fetal lung fibroblasts. The fragment CBSA56/A5P (F8) again showed extensive interactions to

A
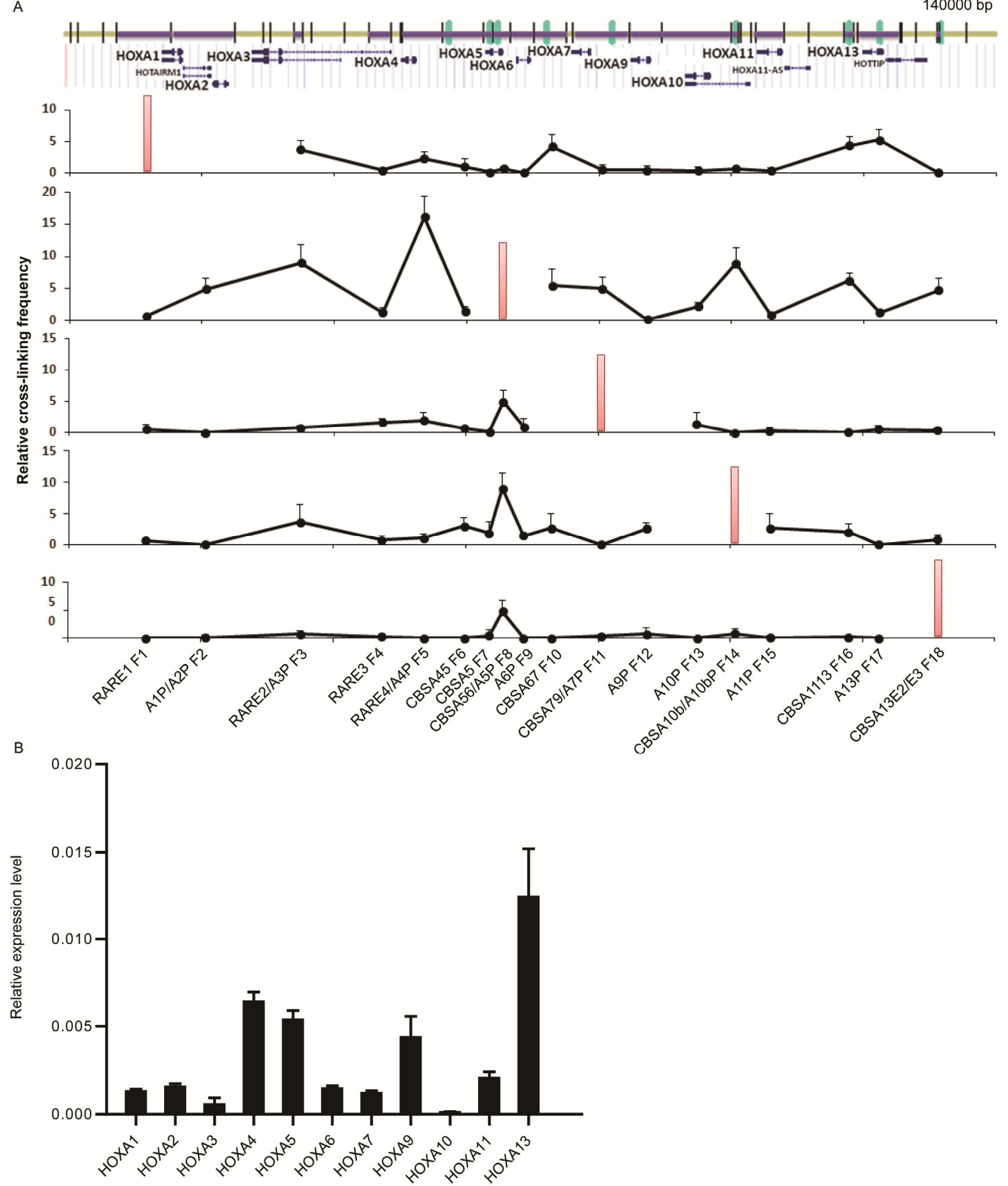

Figure 3 Higher-order chromatin structure of HOXA locus and expression of the HOXA genes in 293T cells. A, The fragment containing CBSA56 showed extensive interactions to other parts of the HOXA locus in $293 \mathrm{~T}$ cells. The crosslinking patterns of five leader fragments are shown. All symbols are the same as in Figure 2. The SEM of the triplicate experiments is indicated. B, Most of HOXA genes were actively transcribed in 293T cells. The HOXA gene expression was determined by quantitative RT-PCR and normalized to GAPDH. Error bars represent standard errors of the means (SEM) of three independent cell cultures. 
the other parts of the HOXA locus. However, the interactions between the leader fragment CBSA56/A5P (F8) and 5' part of this locus were relatively reduced, which is consistent to the activation of $5^{\prime}$ HOXA genes in 293T cells (Figure 3B). These data suggest that the architectural role of CTCF may be a general event in the regulation of HOXA genes.

\subsection{Cohesin is the dynamic regulator of $\mathrm{HOXA}$ cluster higher-order chromatin structure in primary fibroblasts}

Strong CTCF binding were detected at most predicted CTCF binding sites of the HOXA gene cluster, including the CBSA79, CBSA1113 and CBSA13E3. However, CTCF enrichment at all detected CBSs was found comparable in the two types of cells (Figure 4A), or even slightly enhanced at several CBSs in foreskin fibroblasts that display relatively "open" chromatin conformation at the locus. CTCF provides docking sites for co-factors represented by the cohesin complex $[22,23]$. We found that the enrichment of cohesin co-localizes with that of CTCF at most of CBSs, especially at $5^{\prime}$ region of $\mathrm{HOXA}$ locus (Figure 4B). Significant lower cohesin enrichment in human foreskin fibroblasts than that in lung fibroblasts was detected at CBSA5, CBSA79, CBSA1113 and CBSA13E3. The decreased cohesin recruitment is principally consistent with the lower chromatin interactions of the HOXA locus in foreskin fibroblasts, suggesting that cohesin, rather than CTCF, is more likely responsible for the cell-type specific chromatin organization in this gene cluster. Combined with the fact that 5' HOXA genes (HOXA9-HOXA13) were expressed in foreskin fibroblasts other than in lung fibroblasts, cohesin and the CBS-centered higher-order chromatin structure may preferentially play a repressive role in the regulation of $5^{\prime}$ HOXA genes.

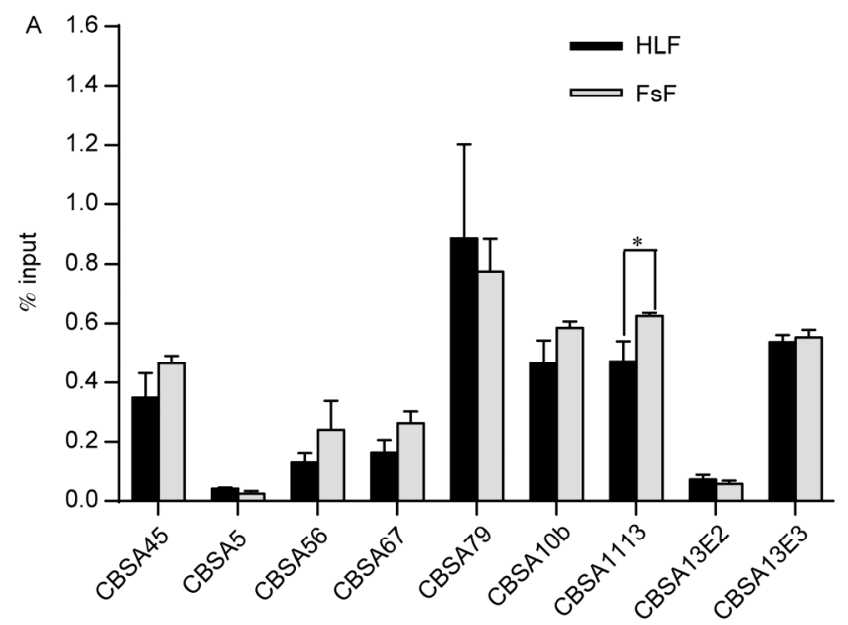

\section{Discussion}

In the present study, we provided the first evidence of the correlation between the CBS-centered chromatin organization and the transcriptional control of HOXA genes from primary human fibroblasts. Our results imply that CTCF and cohesin are involved in chromatin architecture organization and transcriptional control of $H O X A$ cluster in primary fibroblasts, and are potentially important for the formation of spatial collinearity. Our study also suggests that the fetal lung fibroblasts and foreskin fibroblasts of complementary HOXA expression pattern provide ideal models for studying the spatial collinearity of this gene cluster.

Increasing evidences emphasis that CTCF primarily acts to mediate loop formation by its ability to bind DNA and self-associate or associate with subnuclear sites [24,25]. Whether CTCF exerts repressive or active influence on transcription is dependent on the genomic context of the endogenous locus. Cohesin complexes are frequently found to be associated with CTCF in genome organization and are usually responsible for the formation of cell-type specific association between the CBSs [26]. In the two types of human primary fibroblasts, we observed differential enrichment of the cohesin protein RAD21 at the CBSs within HOXA locus, instead of significant changes in CTCF binding signals between the two cell types. The more intensive cohesin recruitment corresponds with the more "close" chromatin conformation in the fetal lung fibroblasts, compared with that in the foreskin fibroblasts.

While the CBSA79 that resides in between HOXA7 and HOXA9 genes shows the highest signal of $\mathrm{CTCF} /$ cohesin binding in the HOXA locus (Figure 4), 3C assay showed that the CBSA56 of weak CTCF/cohesin binding exhibits the most remarkable interactions with both $3^{\prime}$ and $5^{\prime}$ parts of the HOXA locus in both types of fibroblasts. The relatively

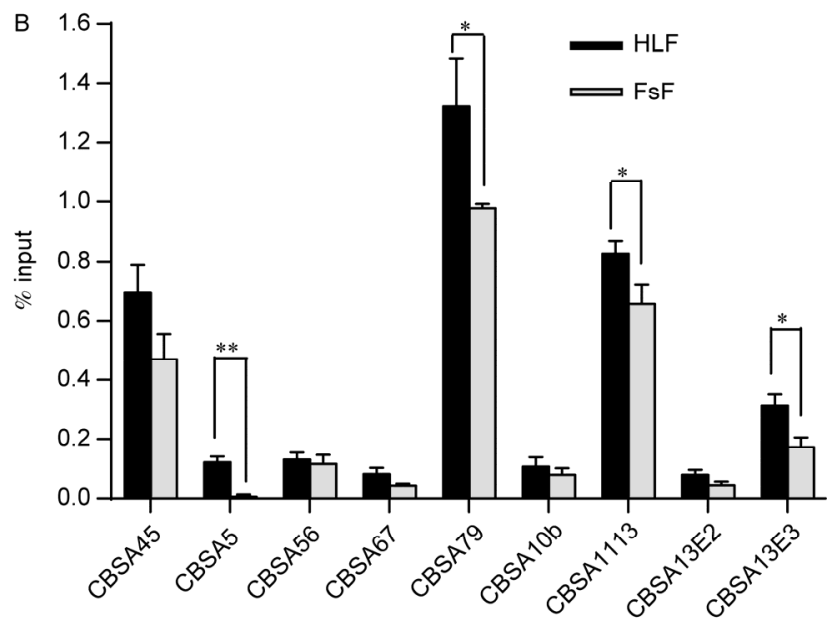

Figure 4 CTCF enrichment over HOXA locus was comparable in human fetal lung fibroblasts (HLF) and foreskin fibroblasts (FsF), while cohesin showed lower enrichment in foreskin fibroblasts. ChIP assays were performed using whole-cell extracts from human lung and foreskin fibroblasts with antibodies against CTCF (A) or cohesin protein RAD21 (B). The enrichment is shown as a percentage of the input. The level of IgG-enriched DNA was negligible and is not shown. The error bars represent SEM. The experiments were performed in triplicate. **, $P<0.01 ; *, P<0.05$ (student's $t$-test). 
weak $\mathrm{CTCF} /$ cohesin recruitment at CBSA56 therefore implies the involvement of other co-factors or architectural proteins in mediating the frequent chromatin contacts between CBSA56 and other sites on the gene cluster.

Our data support a role of $\mathrm{CTCF} /$ cohesin in chromatin architectural and transcriptional control of HOXA cluster in human primary fibroblasts. Further single cell analysis and targeted genome editing strategy will be required to reveal in great detail the dynamic chromatin interactions and the causal relationship between cell-type specific chromatin organization, epigenomics and transcriptional regulation of the locus in the primary cells. Fine mapping of HOXA cluster chromatin interactions in additional cell systems will help identify more cis-elements and regulatory proteins that mediate either the conserved or the cell type-specific contacts in the gene locus.

The authors declare that they have no conflict of interest.

This work was supported by the National Natural Science Foundation of China (31030026), the National Basic Research Program (2011CB965203), and the PUMC Youth funds (3332013138). We are grateful to Dr Zhao Na from Skin Laboratory of Peking Union Medical College Hospital for kindly providing the human foreskin fibroblasts.

1 Rinn JL, Bondre C, Gladstone HB, Brown PO, Chang HY. Anatomic demarcation by positional variation in fibroblast gene expression programs. PLoS Genet, 2006, 2: e119

2 Chang HY, Chi JT, Dudoit S, Bondre C, van de Rijn M, Botstein D, Brown PO. Diversity, topographic differentiation, and positional memory in human fibroblasts. Proc Natl Acad Sci USA, 2002, 99: 12877-12882

3 Wang KC, Helms JA, Chang HY. Regeneration, repair and remembering identity: the three Rs of Hox gene expression. Trends Cell Biol, 2009, 19: 268-275

4 Duboule D. The rise and fall of Hox gene clusters. Development, 2007, 134: 2549-2560

5 Spitz F, Gonzalez F, Duboule D. A global control region defines a chromosomal regulatory landscape containing the HoxD cluster. Cell, 2003, 113: 405-417

6 Sharpe J, Nonchev S, Gould A, Whiting J, Krumlauf R. Selectivity, sharing and competitive interactions in the regulation of Hoxb genes. EMBO J, 1998, 17: 1788-1798

7 Kmita M, Duboule D. Organizing axes in time and space; 25 years of colinear tinkering. Science, 2003, 301: 331-333

8 Kondo T, Duboule D. Breaking colinearity in the mouse HoxD complex. Cell, 1999, 97: 407-417

9 Roelen BA, de Graaff W, Forlani S, Deschamps J. Hox cluster polarity in early transcriptional availability: a high order regulatory level of clustered Hox genes in the mouse. Mech Dev, 2002, 119: 81-90
10 Fraser J, Rousseau M, Shenker S, Ferraiuolo MA, Hayashizaki Y, Blanchette M, Dostie J. Chromatin conformation signatures of cellular differentiation. Genome Biol, 2009, 10: R37

11 Rousseau M, Crutchley JL, Miura H, Suderman M, Blanchette M, Dostie J. Hox in motion: tracking HoxA cluster conformation during differentiation. Nucleic Acids Res, 2014, 42: 1524-1540

12 Chambeyron S, Bickmore WA. Chromatin decondensation and nuclear reorganization of the HoxB locus upon induction of transcription. Genes Dev, 2004, 18: 1119-1130

13 Noordermeer D, Leleu M, Splinter E, Rougemont J, De Laat W, Duboule D. The dynamic architecture of Hox gene clusters. Science, 2011, 334: 222-225

14 Chambeyron S, Da Silva NR, Lawson KA, Bickmore WA. Nuclear re-organisation of the Hoxb complex during mouse embryonic development. Development, 2005, 132: 2215-2223

15 Phillips JE, Corces VG. CTCF: master weaver of the genome. Cell, 2009, 137: 1194-1211

16 Parelho V, Hadjur S, Spivakov M, Leleu M, Sauer S, Gregson HC, Jarmuz A, Canzonetta C, Webster Z, Nesterova T, Cobb BS, Yokomori K, Dillon N, Aragon L, Fisher AG, Merkenschlager M. Cohesins functionally associate with CTCF on mammalian chromosome arms. Cell, 2008, 132: 422-433

17 Gause M, Schaaf CA, Dorsett D. Cohesin and CTCF: cooperating to control chromosome conformation? Bioessays, 2008, 30: 715-718

18 Xu M, Zhao GN, Lv X, Liu G, Wang LY, Hao DL, Wang J, Liu DP, Liang CC. CTCF controls HOXA cluster silencing and mediates PRC2-repressive higher-order chromatin structure in NT2/D1 cells. Mol Cell Biol, 2014, 34: 3867-3879

19 Li M, Ti D, Han W, Fu X. Microenvironment-induced myofibroblastlike conversion of engrafted keratinocytes. Sci China Life Sci, 2014, 57: 209-220

20 Liu X, Huang Q, Li F, Li CY. Enhancing the efficiency of direct reprogramming of human primary fibroblasts into dopaminergic neuron-like cells through p53 suppression. Sci China Life Sci, 2014, 57: $867-875$

21 Rinn JL, Kertesz M, Wang JK, Squazzo SL, Xu X, Brugmann SA, Goodnough LH, Helms JA, Farnham PJ, Segal E, Chang HY. Functional demarcation of active and silent chromatin domains in human HOX loci by noncoding RNAs. Cell, 2007, 129: 1311-1323

22 Rubio ED, Reiss DJ, Welcsh PL, Disteche CM, Filippova GN, Baliga NS, Aebersold R, Ranish JA, Krumm A. CTCF physically links cohesin to chromatin. Proc Natl Acad Sci USA, 2008, 105: 8309-8314

23 Kim YJ, Cecchini KR, Kim TH. Conserved, developmentally regulated mechanism couples chromosomal looping and heterochromatin barrier activity at the homeobox gene a locus. Proc Natl Acad Sci USA, 2011, 108: 7391-7396

24 Yusufzai TM, Felsenfeld G. The 5'-HS4 chicken beta-globin insulator is a CTCF-dependent nuclear matrix-associated element. Proc Natl Acad Sci USA, 2004, 101: 8620-8624

25 Yusufzai TM, Tagami H, Nakatani Y, Felsenfeld G. CTCF tethers an insulator to subnuclear sites, suggesting shared insulator mechanisms across species. Mol Cell, 2004, 13: 291-298

26 Hou C, Dale R, Dean A. Cell type specificity of chromatin organization mediated by CTCF and cohesin. Proc Natl Acad Sci USA, 2010, 107: 3651-3656

Open Access This article is distributed under the terms of the Creative Commons Attribution License which permits any use, distribution, and reproduction in any medium, provided the original author(s) and source are credited. 